\title{
GRK5 deficiency exaggerates inflammatory changes in TgAPPsw mice
}

\author{
Longxuan $\mathrm{Li}^{1}$, Jun Liu ${ }^{1,4}$ and William Z Suo*1,2,3
}

Address: ${ }^{1}$ Lab. for Alzheimer's Disease \& Aging Res., VA Med. Center, Kansas City, MO 64128, USA, ${ }^{2}$ Department of Neurology, Univ. of Kansas Med. Center, Kansas City, KS 66170, USA, ${ }^{3}$ Department of Molecular \& Integrative Physiology, Univ. of Kansas Med. Center, Kansas City, KS 66170, USA and ${ }^{4}$ Department of Neurology, The 2nd Affiliated Hospital, Sun Yat-sen University, Guangzhou, 510120, PR China

Email: Longxuan Li - longxuanlee2006@yahoo.com; Jun Liu - docliujun@hotmail.com; William Z Suo* - Zhiming.William@va.gov

* Corresponding author

Published: 3 June 2008

Journal of Neuroinflammation 2008, 5:24 doi:10.1 186/1742-2094-5-24
Received: 28 March 2008

Accepted: 3 June 2008

This article is available from: http://www.jneuroinflammation.com/content/5/I/24

(C) 2008 Li et al; licensee BioMed Central Ltd.

This is an Open Access article distributed under the terms of the Creative Commons Attribution License (http://creativecommons.org/licenses/by/2.0), which permits unrestricted use, distribution, and reproduction in any medium, provided the original work is properly cited.

\begin{abstract}
Background: Deficiency of membrane G-protein coupled receptor (GPCR) kinase-5 (GRK5) recently has been linked to early $A D$ pathogenesis, and has been suggested to contribute to augmented microglial activation in vitro by sensitizing relevant GPCRs. However, GRK5 deficient mice did not show any signs of microgliosis, except for their moderate increase in axonal defects and synaptic degenerative changes during aging. We have speculated that one possible reason for the absence of microgliosis in these animals might be due to lack of an active inflammatory process involving activated GPCR signaling, since GRKs only act on activated GPCRs. The objective of this study was to determine whether the microgliosis is exaggerated in TgAPPsw (Tg2576) mice also deficient in GRK5, in which fibrillar $\beta$-amyloid $(A \beta)$ and an active inflammatory process involving activated GPCR signaling are present.
\end{abstract}

Methods: Both quantitative and qualitative immunochemistry methods were used to evaluate the microgliosis and astrogliosis in these animals.

Results: We found that inactivation of one copy of the GRK5 gene in the TgAPPsw mice resulted in approximately doubled extent of microgliosis, along with significantly exaggerated astrogliosis, in both hippocampus and cortex of the aged animals. Consistent with previous observations, the activated microglia were located primarily near or surrounding the fibrillar $A \beta$ deposits.

Conclusion: The results demonstrate that GRK5 deficiency in vivo significantly exaggerates microgliosis and astrogliosis in the presence of an inflammatory initiator, such as the excess fibrillar $A \beta$ and the subsequent active inflammatory reactions in the TgAPPsw mice.

\section{Background}

Alzheimer's disease (AD) is a devastating neurodegenerative disorder affecting a growing number among the aging population. Although its etiology remains to be elucidated, pathological evidence indicates that, in addition to the characteristic senile plaques and neurofibrillary tangles, significant brain inflammatory changes, featured with exaggerated gliosis and increased proinflammatory cytokines, are an important component of the pathology $[1,2]$.

Inflammation is a double-edged sword. For instance in $\mathrm{AD}$, an appropriate degree of inflammatory reaction may be beneficial for clearance of aggregated proteins and/or 
degenerated cells. However, if the inflammation is overly reactive, the secondary inflammatory neuronal damage may become a driving force for the disease, as appears to be the case in $\mathrm{AD}$ [1]. Therefore, it is important to understand what factors may exaggerate the brain inflammation in $\mathrm{AD}$ so that corresponding therapeutic approaches can be developed to ameliorate such changes.

The extent of inflammatory reactions may be regulated by a variety of factors, such as proinflammatory cytokines, chemokines, complement systems, and others. In $\mathrm{AD}$, fibrillar $\beta$-amyloid $(A \beta)$ has been shown to directly activate microglial cells and result in increased levels of proinflammatory cytokines, such as interleukin- 1 beta (IL-1 $\beta$ ) and tumor necrosis factor- $\alpha$ [1-3]. In addition, it can activate the complement system to generate $\mathrm{C} 3 \mathrm{a}$ and $\mathrm{C} 5 \mathrm{a}$ [4]. Moreover, fibrillar $\mathrm{A} \beta$ accumulation is associated with increased levels of many chemokines and chemokine receptors, such as monocyte chemoattractant protein-1 (CCR2 ligand), macrophage inflammatory protein 1 (CCR3 ligand), interferon-inducible protein-10 (CXCR3 ligand), IL-8 (ligand for CXCXR1 and CXCR2) and CCR1/ 3/5. [5-7]. Amongst the inflammatory regulators associated with $\mathrm{AD}$, there is one large category that exerts their cellular effects through heterotrimeric G-protein-coupled receptors (GPCRs). These include all the chemokines [6] and the anaphylatoxins C3a and C5a $[8,9]$.

In a recent study, we showed that deficiency of membrane GPCR kinase-5 (GRK5) occurs during early $\mathrm{AD}$, and the GRK5 deficiency contributes to augmented microglial activation in vitro via impaired sensitization of relevant GPCRs [10]. GRK5 is one of seven GRK family members, whose primary function is to desensitize activated GPCRs by phosphorylating the activated receptor to promote uncoupling of receptor-dependent G-protein activation and by initiating receptor internalization $[11,12]$. Therefore, the lack of membrane (functional) GRK5 resulted in prolonged signaling and enhanced microglial activation, which well explained the phenomena that we observed in the cultured microglial cells [10]. Moreover, we speculated that the same phenomena might also take place in vivo. However, our later study in GRK5 deficient mice (GRK5KO), an animal model resembling the functional GRK5 deficiency in $\mathrm{AD}$, failed to show any sign of microgliosis, except for moderate increase in axonal defects and synaptic degenerative changes in these animals [13]. The negative in vivo data appeared to be inconsistent with our earlier in vitro data. Nonetheless, a hallmark of GRK action is to function only after GPCRs are activated $[11,12]$. In other words, whether or not an impact of GRK5 deficiency on a particular stimulus can be revealed depends on whether or not such a stimulus is present. We have reported that, although the GRK5 deficiency indeed caused a mild increase of $A \beta$ level, no fibrillar $A \beta$ deposits or any significant signs of microglial activation were observed in the GRK5KO mice [13]. Therefore, we speculated that one reason for the absence of microgliosis in these animals might be due to lack of inflammatory initiators, such as fibrillar $A \beta$, or any of the downstream chemokines or anaphylatoxins.

In this study, we crossbred the GRK5KO mice with TgAPPsw (Tg2576) mice. TgAPPsw mice overexpress human $\beta$-amyloid precursor protein ( $\beta A P P)$ carrying Swedish double mutations, and have been shown to display fibrillar $\mathrm{A} \beta$ deposition and moderate levels of inflammation [14-16]. Therefore, the resultant TgAPPsw mice deficient in GRK5 should provide an ideal model to determine if our previous speculations are correct, and GRK5 deficiency plays a significant role in enhancing brain inflammation in $\mathrm{AD}$.

\section{Methods \\ Animals}

GRK5KO mice were generated by targeted deletion of exons 7 and 8 of the GRK5 gene, encoding critical sub-elements I through III of the protein kinase catalytic domain, as detailed previously [17]. Heterozygous GRK5KO mice (C57/BL6 background) were bred with TgAPPsw (Tg2576) mice (also with a C57/BL6 background) to produce wild type (WT, APPsw ${ }^{-} / / \mathrm{GRK}^{+/+}, n=6$ ), GRK5KO $\left(\mathrm{APPsw}^{-/} / \mathrm{GRK}^{+/-}, n=6\right)$, TgAPPsw $\left(\mathrm{APPsw}^{+/ /} / \mathrm{GRK}^{+/+}, n\right.$ $=6)$, and the double $\left(\mathrm{APPsw}^{+/-} / \mathrm{GRK}^{+/-}, n=6\right)$ mice for this study. Due to known gender difference [18-20] [Li, 2008, BRB-submitted], only female mice (18-month old) were used in this study. A tail DNA preparation was used for genotyping as described below. All procedures for using these animals were approved by the KCVAMC Institutional Animal Care and Use Committee.

\section{Genotyping}

Mouse-tail DNA was prepared as previously described [13]. Genotyping was performed by polymerase chain reaction (PCR) amplification of the tail genomic DNA with five different primers together: two human APP-specific primers, 5'CGGAGGAGGATGACTCGGAT3' and 5'CAGCTGCTGTCTCTCGTTGG3', which amplify a 500 bp DNA fragment, were used to identify the presence of the human APP transgene; three additional primers specific for WT murine GRK5 (425 bp) and the GRK5KO loci $(200$ bp) (5'CAAGTGTGAGGTAGGGTACAGAAT3', 5'CTATCCATTCACCTCCATGCTCCC3', and 5'AACTCTGGTACAGACAGGATCTCT3') were used to identify the presence of WT or the targeted murine GRK5 gene. The PCR used a $20 \mu \mathrm{l}$ reaction volume containing 1.25 units of Flexi DNA polymerase (Promega, Madison, WI), $200 \mu \mathrm{M}$ dNTPs, $2 \mathrm{mM} \mathrm{MgCl}_{2}$, and $250 \mathrm{nM}$ of each of the APP preimers and $1 \mu \mathrm{M}$ of each of the three GRK5 primers. The amplification protocol entailed 35 cycles of 
denaturation at $94^{\circ} \mathrm{C}$ for $15 \mathrm{~s}$, annealing at $62^{\circ} \mathrm{C}$ for $15 \mathrm{~s}$ and extension at $72^{\circ} \mathrm{C}$ for $15 \mathrm{~s}$, and then followed by a 10 min final extension at $72^{\circ} \mathrm{C}$. The PCR products $(10 \mu \mathrm{l})$ were then analyzed and visualized on $1.5 \%$ agarose gels. Fig. 1 shows an example of a typical genotyping result including the four different genotypes used in this study.

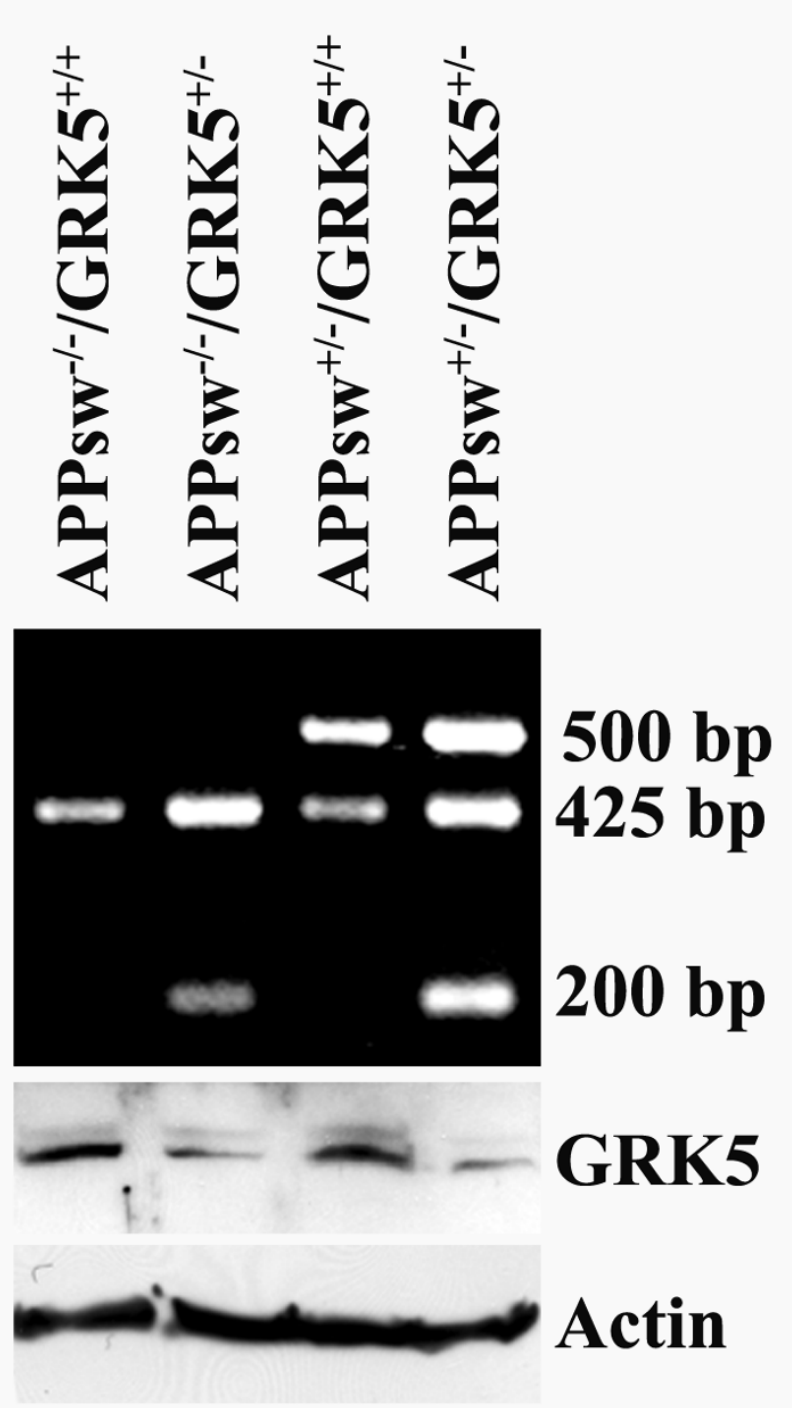

Figure I

Genotypes and GRK5 expression in TgAPPsw mice with reduced GRK5. Top panel, an example of the genotyping results for the WT (APPsw ${ }^{-/ /} / \mathrm{GRK}^{+/+}$), heterozygote GRK5KO (APPsw - $^{-/} / \mathrm{GRK}^{+/-}$), TgAPPsw (APPsw ${ }^{+/-/ G R K 5^{+/}}$

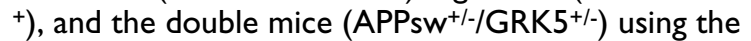
ascribed five primers. 500 base pair (bp) band, human APPsw; 425 bp band, WT GRK5; 200 bp band, inactivated GRK5. Bottom panels are representative Western blots for GRK5 and $\beta$-actin (internal control for the amounts of sample loaded), respectively, as indicated.

\section{Tissue preparations}

All animals were anesthetized and perfused with cold phosphate buffered saline (PBS), their brains removed, and left hemispheres were post-fixed with $4 \%$ paraformaldehyde and processed for pathology. To minimize experimental variations, multi-brain embedding and sectioning techniques were adapted according to previously published procedures [13]. Briefly, twenty-four left hemispheres (including six from each genotype) were embedded into six blocks, with each block containing one hemisphere from each of the WT, GRK5KO, APP and the double mice, respectively. The multi-brain embedding allows the subsequent sectioning and staining procedures for all samples in a single block to be performed in a unified condition, which reduces variations that often occur when each brain is manipulated separately. Frozen sections were taken at a thickness of $20 \mu \mathrm{m}$ in the coronal plane through the hemispheres. All sections were collected sequentially into eight groups with $160 \mu \mathrm{m}$ intervals.

\section{Immunofluorescent (IF) staining}

IF staining was performed on the multi-brain sections as previously described [13]. The only difference was the target antibody used: mouse monoclonal antibody (mAb) to human $A \beta$ (Alpha Diagnostics International, San Antonio, TX; 1:600), rat mAb to CD45 (MCA1388, Clone: IBL3/16, Serotec, Raleigh, NC; 1:25), rat mAb to mouse CD11b (Mac-1, Clone: M1/70.15, Serotec, Raleigh, NC; 1:25), mouse mAb to CD11b/c (Clone MRC OX-42, Serotec, Raleigh, NC; 1:25), rabbit polyclonal antibody (pAb) to human IL-1 $\beta$ (Santa Cruz Biotechnology, Santa Cruz, CA; $1: 25)$, mAb to glial fibrillary acidic protein (GFAP) conjugated to Cy3 (Sigma, St. Louis, MO;1:200), or rabbit pAb to IL-6 (Pierce Biotechnology, Rockford, IL; 1:100). DAPI (Invitrogen Corporation, Carlsbad, CA) was used to detect nuclei.

\section{Image Analysis}

For quantitative image analysis, serial coronal sections taken from 1.70 to $3.16 \mathrm{~mm}$ posterior to the bregma were examined for $\mathrm{CD}_{4} 5^{+}$microglia and $\mathrm{GFAP}^{+}$astrocytes. Six animals from each group were evaluated. For each animal, antigens were detected in 5 parallel multi-brain sections having a defined distance of $160 \mu \mathrm{m}$ and showing both the hippocampus and cortex. In each section, the hippocampus and the cortex were evaluated for the number of stained cells, and integral staining density (sum of all individual optical densities of each pixel in the area being measured). Images were acquired as digitized tiff files to retain maximal resolution using a Leica DMI $6000 \mathrm{~B}$ microscope microscope at $100 \times$ final magnification with an attached digital camera system (Leica DFC 340 FX, Heerbrugg, Germany). The digital images were then routed into a Windows PC for quantitative analyses using 
Image-Pro Plus software, with the examiner blinded to sample identifiers. To maintain consistency across animals, a rectangular box $(0.42 \times 0.26 \mathrm{~mm})$ was centered over the area of interest. Except for fewer sections used, all other quantifying procedures were the same as stereological quantification. The final results were shown as number of immunopositive cells or immunoreactivity per $\mathrm{mm}^{2}$.

\section{Western blot}

At euthanasia, the cold PBS-perfused right hemispheres were further dissected into anterior cortex, posterior cortex, striatum, hippocampus, brainstem, and cerebellum. Protein extract preparation, total protein content determination, western blotting, and semi-quantitative analyses were performed as previously described $[10,13]$. Primary Abs used were: rabbit pAb to GRK5 (Santa Cruz Biotechnology, Santa Cruz, CA; 1:200) and mAb to $\beta$-actin (Sigma, St. Louis, MO; 1:1000).

\section{Statistics}

Data were expressed as the mean \pm S.E.M, and analyzed by ANOVA using Prism 4.3 (GraphPad Software, San Diego, CA). Post-hoc comparisons of means were made using Bonferroni's method with significance set at 0.05 .

\section{Results}

In order to determine whether GRK5 deficiency has an impact on brain inflammation in $\mathrm{AD}$, we crossbred the TgAPPsw mice with the GRK5KO mice and generated the TgAPPsw mice deficient in GRK5. Only heterozygotes were used in this study, which included four genotype groups: WT control (APPsw-//GRK5+/+), GRK5KO heterozygote control (APPsw ${ }^{-/} / \mathrm{GRK}^{+/}$), TgAPPsw $\left(\mathrm{APPsw}^{+/-}\right.$$\mathrm{GRK}^{+/+}$) and the double (APPsw ${ }^{+/-} / \mathrm{GRK}^{+/-}$) mice. As shown in Fig. 1, inactivation of one copy of the GRK5 gene resulted in at least $50 \%$ down-regulation of the GRK5 expression at the protein level in the heterozygote GRK5KO and the double mice.

Evaluation of potential changes in brain inflammation was performed in 18-month old female mice, due to the known gender impact in these mice [18-20] [Li, 2008, BRB-submitted]. Immunofluorescent staining with antibodies against microglial and astrocyte markers was employed to reveal changes in microgliosis and astrogliosis. Our initial examination with antibody against CD45, a surface marker of microglia [15], revealed an increase of microgliosis in both hippocampal (Fig. 2A-D) and cortical (Fig. 2E-H) areas of the double mice as compared to the TgAPPsw mice. Subsequent characterization of the microgliosis showed that, consistent with previous findings [15], CD45+ microglial cells were largely located surrounding or near $\mathrm{A} \beta$ deposits (Fig. 3A-C). Staining with a rat $\mathrm{mAb}$ to $\mathrm{CD} 11 \mathrm{~b}$ (Mac-1), another well-established microglial and macrophage marker $[15,21]$, revealed sim-
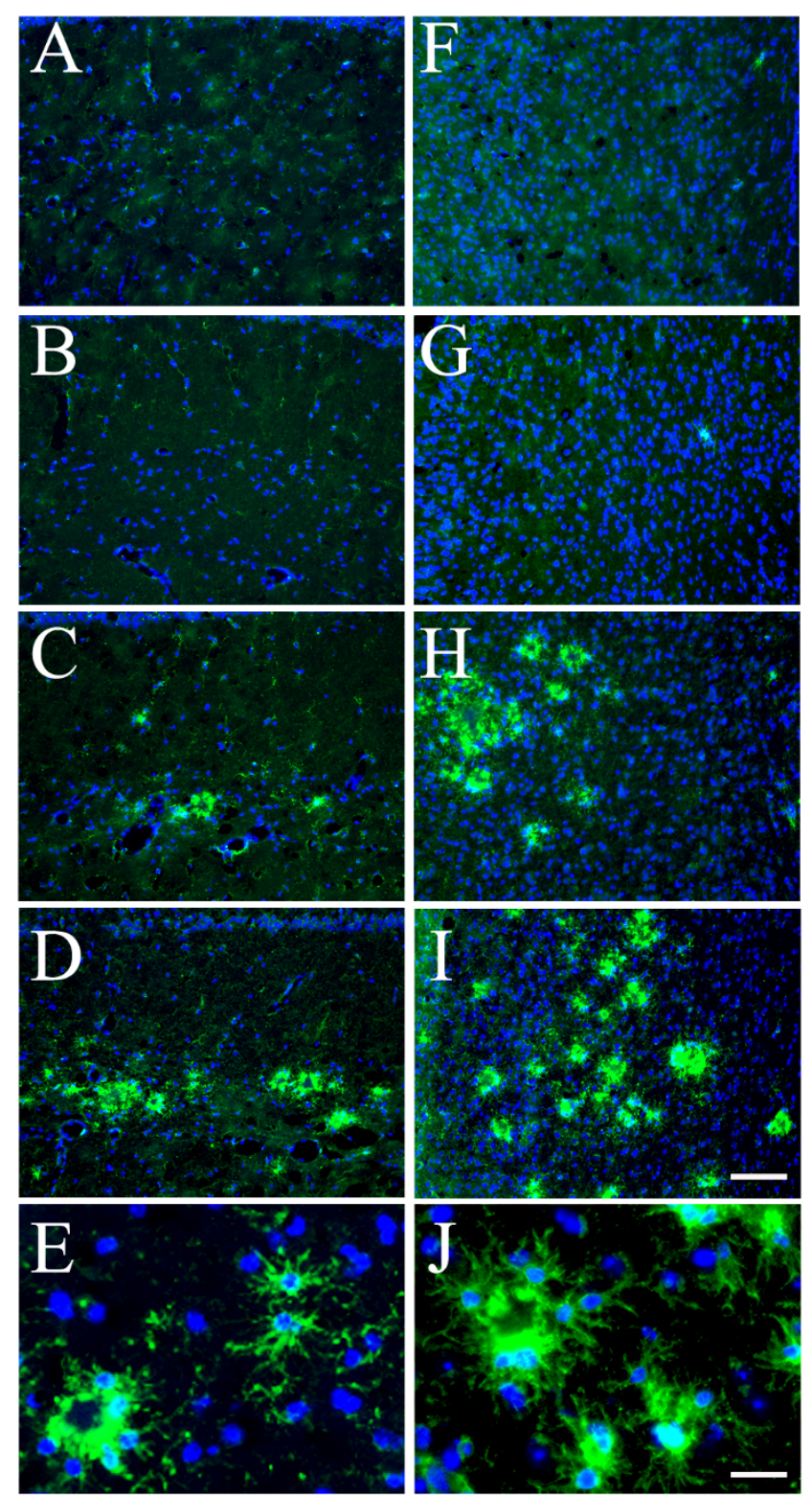

Figure 2

Increased CD45 ${ }^{+}$microglial cells in hippocampus and cortex of the TgAPPsw mice with reduced GRK5.

Representative IF results with anti-CD45 staining (green) in hippocampus (A-D) and cortex (F-I) of WT (A \&F), heterozygote GRK5KO (B \&G), TgAPPsw (C \&H), and the double mice (D \& I), respectively. Scale bar in panel I is for panels A-D and F-I: $100 \mu \mathrm{m}$. Panels E \&J, examples of high magnification views of $\mathrm{CD} 45^{+}$-microglial cells in hippocampus (E) and cortex $(\mathrm{J})$ of the double mice that show details of activated microglial morphology. Scale bar in panel J is for panels E \&J: $20 \mu \mathrm{m}$. Blue indicates reference DAPI staining of nuclei.

ilar changes of microglia in these animals (Fig. 3D-F). In addition, a mouse mAb OX-42 to CD11b/c also decorated the activated microglial cells in a way similar to anti-CD45 

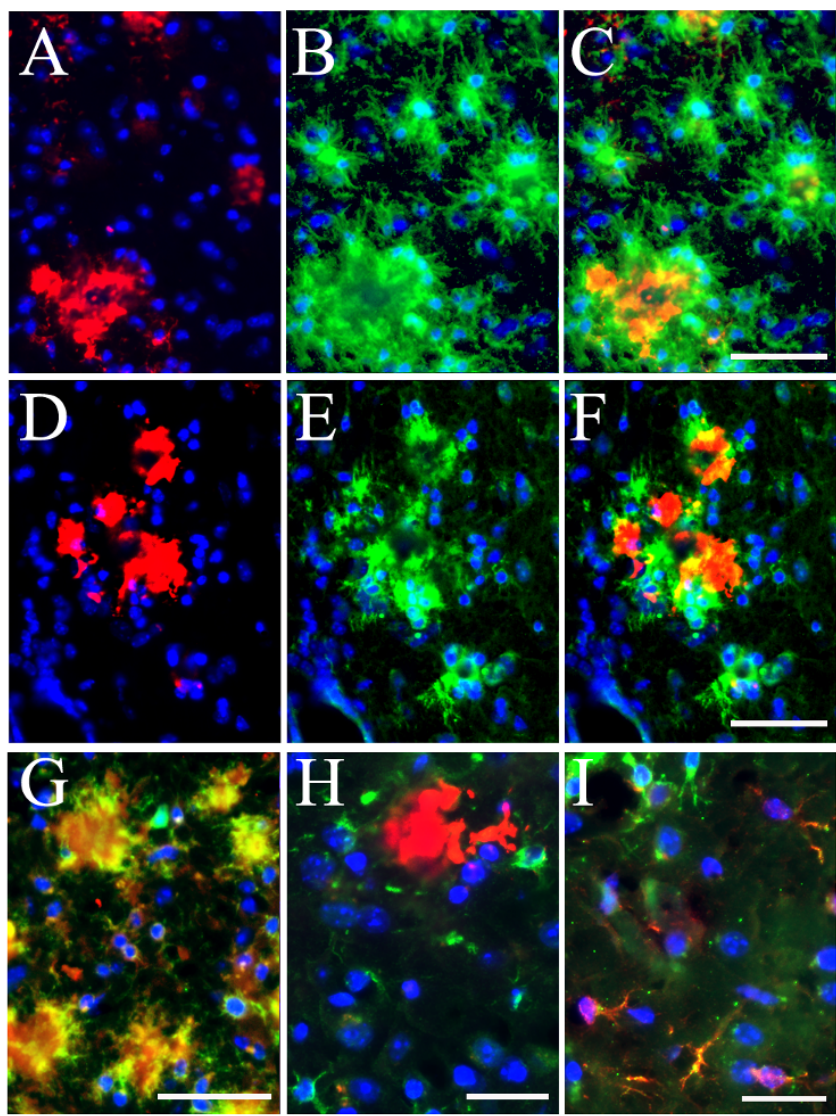

Figure 3

Colocalization of activated microglial cells with $A \beta$ plaques and IL-I $\beta$ immunoreactivity in the TgAPPsw mice with reduced GRK5. Panels A-C, IF staining of $A \beta^{+}$ plaques $\left(\mathbf{A}\right.$, red) and surrounding $C D 45^{+}$microglial cells $(\mathbf{B}$, green), as well as their merged view (C) in the double mice. Scale bar: $50 \mu \mathrm{m}$ for panels A-C. Panels D-F, IF staining of $A \beta^{+}$plaques (D, red) and surrounding $C D I I b^{+}$microglial cells (E, green), as well as their merged view $(\mathbf{F})$ in the double mice. Scale bar: $50 \mu \mathrm{m}$ for panels $\mathbf{D}-\mathbf{F}$. Panel $\mathbf{G}$, an example of merged view for CD45 (green) and CDI Ib/c (clone OX42, red) co-staining of the microglial cells. The image showed that, at least in this particular experimental paradigm, the CD45 antibody stained more specifically for the microglial cell profiles; while the OX42 antibody, in addition to its positive staining of the microglial cells, also non-specifically decorated the plaques. Scale bar: $45 \mu \mathrm{m}$. Panel $\mathbf{H}$, an example of merged view for $A \beta^{+}$plaques (red) and surrounding IL-I $\beta$ immunoreactivity (green) in the double mice. Scale bar: $30 \mu \mathrm{m}$. Panel I, colocalization of $\mathrm{CD} 45^{+}$microglial cells (green) with IL-I $\beta$ immunoreactivity (red) in the double mice. Scale bar: $30 \mu \mathrm{m}$. Blue indicates reference DAPI staining of nuclei.

(Fig. 3G). However, at least in our experiments, OX-42 appeared to non-specifically stain the $A \beta$ plaques, in addition to its positive staining for microglial cells. It has been previously established that activated microglial cells, at least at the early phase, produce IL-1 $\beta$, an important proinflammatory cytokine that participates in a self-sustained vicious cycle to amplify the inflammatory neuronal damage [2]. Our examination revealed that, although less profound than CD45 immunoreactivity (IR), IL-1 $\beta$-IR was indeed found near or surrounding the $A \beta$ plaques (Fig. $3 \mathrm{H}$ ) and was colocalized with $\mathrm{CD} 45^{+}$microglial cells (Fig. 3I). Therefore, the microgliosis in the double mice displayed similar traits to that observed in other AD transgenic models, except for the exaggerated extent.

To better understand the extent of the exaggerated microgliosis in these animals, we quantified CD $45^{+}$microglial cells per $\mathrm{mm}^{2}$ in hippocampus and cortex. Our quantitative image analysis (Fig. 4A \&4B) showed that, as compared to the WT mice, both the TgAPPsw and the double mice displayed significantly more CD $45^{+}$microglial cells in both of the examined brain regions ( $\mathrm{p}<0.001$ for all); when compared to the TgAPPsw mice, the double mice had approximately double the number of $\mathrm{CD} 45^{+}$microglial cells, in both hippocampus and cortex $(\mathrm{P}<0.001)$. In addition, the heterozygote GRK5KO mice did not show any significant changes in the number of $\mathrm{CD} 45^{+}$microglial cells, as compared to the WT, which is consistent with our previous report for the negative finding of brain inflammation in the GRK5KO mice [13]. When two-way ANOVA was used to analyze interactions between the two gene modifications (over-expression of human APPsw and inactivation of one copy of the murine GRK5 gene), a significance of $\mathrm{p}<0.001$ was revealed. Meanwhile, the changes caused by both gene modifications in the double mice were significantly higher than the sum of the changes caused by APPsw over-expression in TgAPPsw and by GRK5 gene inactivation in GRK5KO mice ( $\mathrm{p}=$ 0.003 for hippocampus and $\mathrm{p}<0.001$ for cortex). These results indicate that the two gene modifications synergistically interact with each other, and both contribute the microgliosis observed in the double mice.

In parallel to the microglial analysis, potential changes of astrogliosis in these animals were also assessed using IF staining with antibody to GFAP, an astrocyte-specific intermediate filament protein. We found more hypertrophic astrocytes brightly stained with anti-GFAP in both hippocampus and cortex from the double mice as compared to the TgAPPsw mice, and both the double mice and the TgAPPsw mice showed more hypertrophic astrocytes than the WT or heterozygous GRK5KO mice (Fig. $5 \mathrm{~A}-\mathrm{H})$. Further characterization of the astrogliosis in the double mice revealed that although some of the hypertrophic astrocytes located near $\mathrm{A} \beta$ plaques (Fig. 6A-C), compared to the relation of microglia to $A \beta$ plaques, hypertrophic astrocytes were more widespread in the entire brain region. In addition, at least some of the reactive astrocytes were IL-6 positive (Fig. 6D-F). Quantitative 

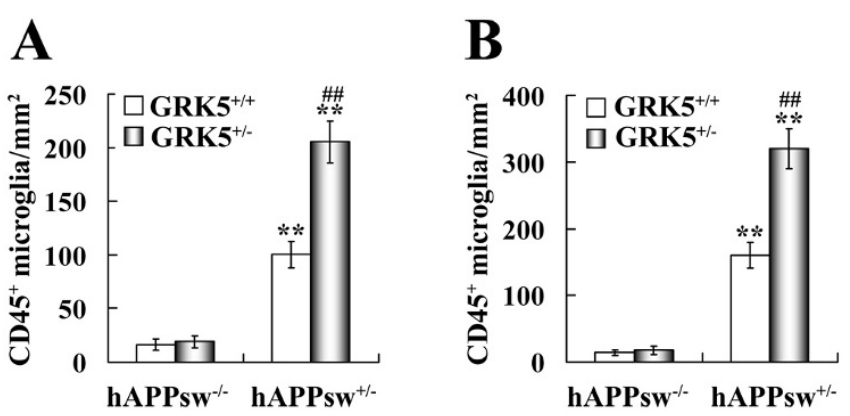

\section{Figure 4}

Quantification of CD45+ microglial cells in hippocampus and cortex of the TgAPPsw mice with reduced GRK5. The numbers of CD45+ microglial cells in hippocampus (A) and cortex (B) of WT, heterozygote GRK5KO, TgAPPsw, and the double mice were quantified as described in methods. Two way-ANOVAs revealed significant interactions between APPsw and GRK5 gene modifications for both hippocampus and cortex $(\mathrm{P}<0.00 \mathrm{I}$ for both regions). The results for Bonferroni post-hoc comparisons of the means were shown as indicated. $* * \mathrm{P}<0.001$ vs WT or heterozygote GRK5KO, \#\# < 0.001 vs TgAPPsw mice.

image analysis (Fig. 7A \&7B) indicated that both the TgAPPsw and the double mice showed significantly more $\mathrm{GFAP}^{+}$astrocytes in the hippocampus and cortex (p < 0.001 for all) as compared to either the WT or the heterozygous GRK5KO mice; furthermore, the double mice displayed greater numbers of astrocytes than that in the TgAPPsw mice ( $\mathrm{p}<0.01$ for hippocampus and $\mathrm{p}<0.001$ for cortex). Consistent with our previous report [13], the number of $\mathrm{GFAP}^{+}$astrocytes was not significantly different between the heterozygous GRK5KO and the WT mice. When two-way ANOVA was employed, significant interactions ( $p<0.05$ for hippocampus, and $\mathrm{p}<0.01$ for cortex) were revealed between the two gene modifications. The comparisons of effects of the double gene modifications with the sum of the effects of the each single gene modification alone revealed significant difference for the cortex $(\mathrm{p}=0.02)$ but not for the hippocampus $(\mathrm{p}=0.10)$.

It is known that both resting and reactive astrocytes express GFAP, but the latter usually expresses higher level of GFAP, and therefore stain brighter. Because of this, counting the number of GFAP+ astrocytes may not accurately reflect the extent of the astrogliosis. Therefore, a quantitative image analysis of GFAP-IR intensity was also performed in parallel to the GFAP+ cell counting. The results were similar to those from simple cell number counting, except that the significance was more striking (Fig. 7C \&7D). The double gene modification effects were significantly higher than the sum of the each single gene alone for both hippocampus $(\mathrm{p}=0.006)$ and cortex $(\mathrm{p}=$
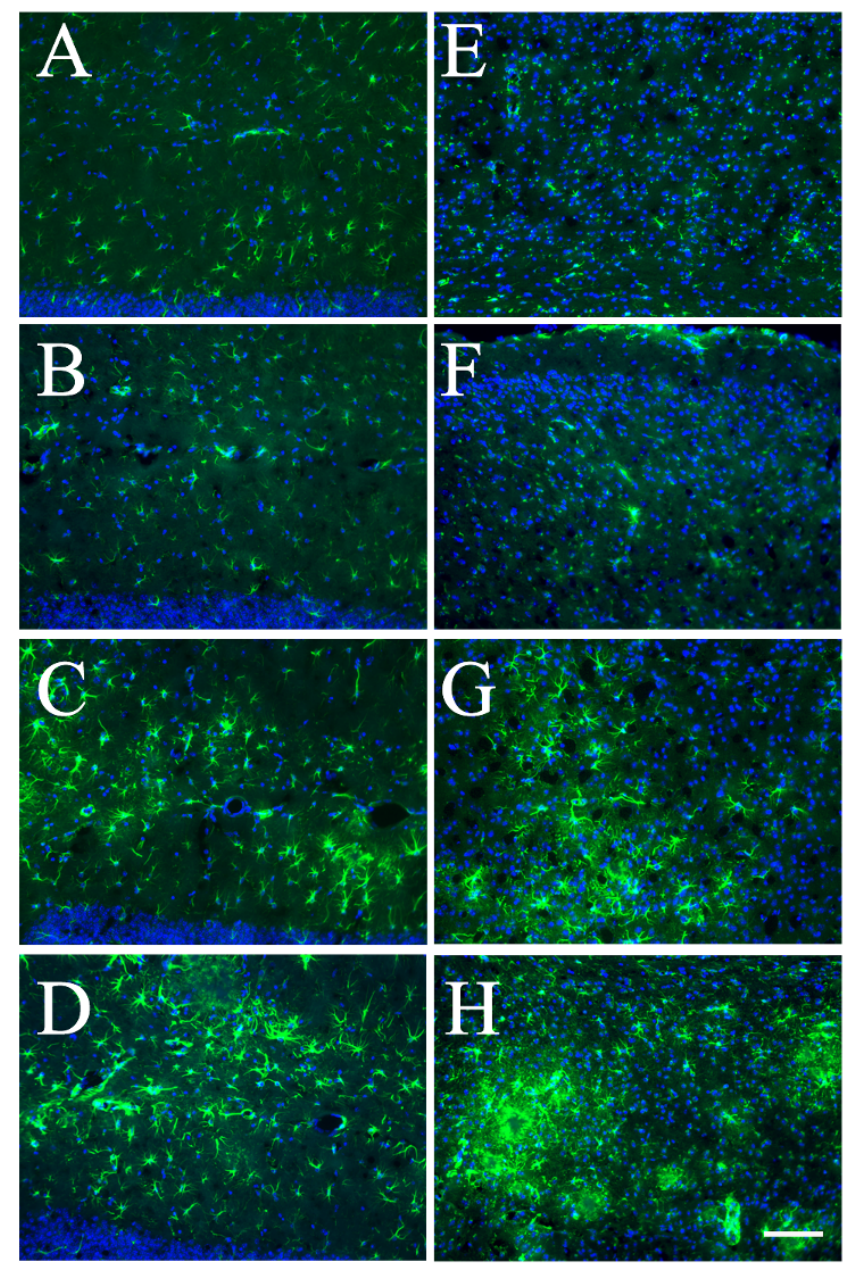

Figure 5

Increased GFAP+ astrocytes in hippocampus and cortex of the TgAPPsw mice with reduced GRK5. Representative IF results with anti-GFAP staining (green) in hippocampus (A-D) and cortex (E-H) of WT (A \&E), heterozygote GRK5KO (B \&F), TgAPPsw (C \&G), and the double mice (D \&H), respectively. Blue indicates reference DAPI staining of nuclei. Scale bar: $100 \mu \mathrm{m}$.

0.009). The interactions between the two gene modifications were also highly significant for both brain regions ( $\mathrm{p}$ $=0.002$ for hippocampus, and $p=0.005$ for cortex). Therefore, these results suggest that Swedish mutated APP gene over-expression and GRK5 deficiency synergistically interact with each other to promote the astrogliosis observed in the double mice.

\section{Discussion}

By analyzing the extent of gliosis in the brains of TgAPPsw mice with normal or reduced GRK5 expression, we demonstrated that the TgAPPsw mice with reduced GRK5 displayed significantly exaggerated microgliosis and astrogliosis. Consistent with our previous report [13], 

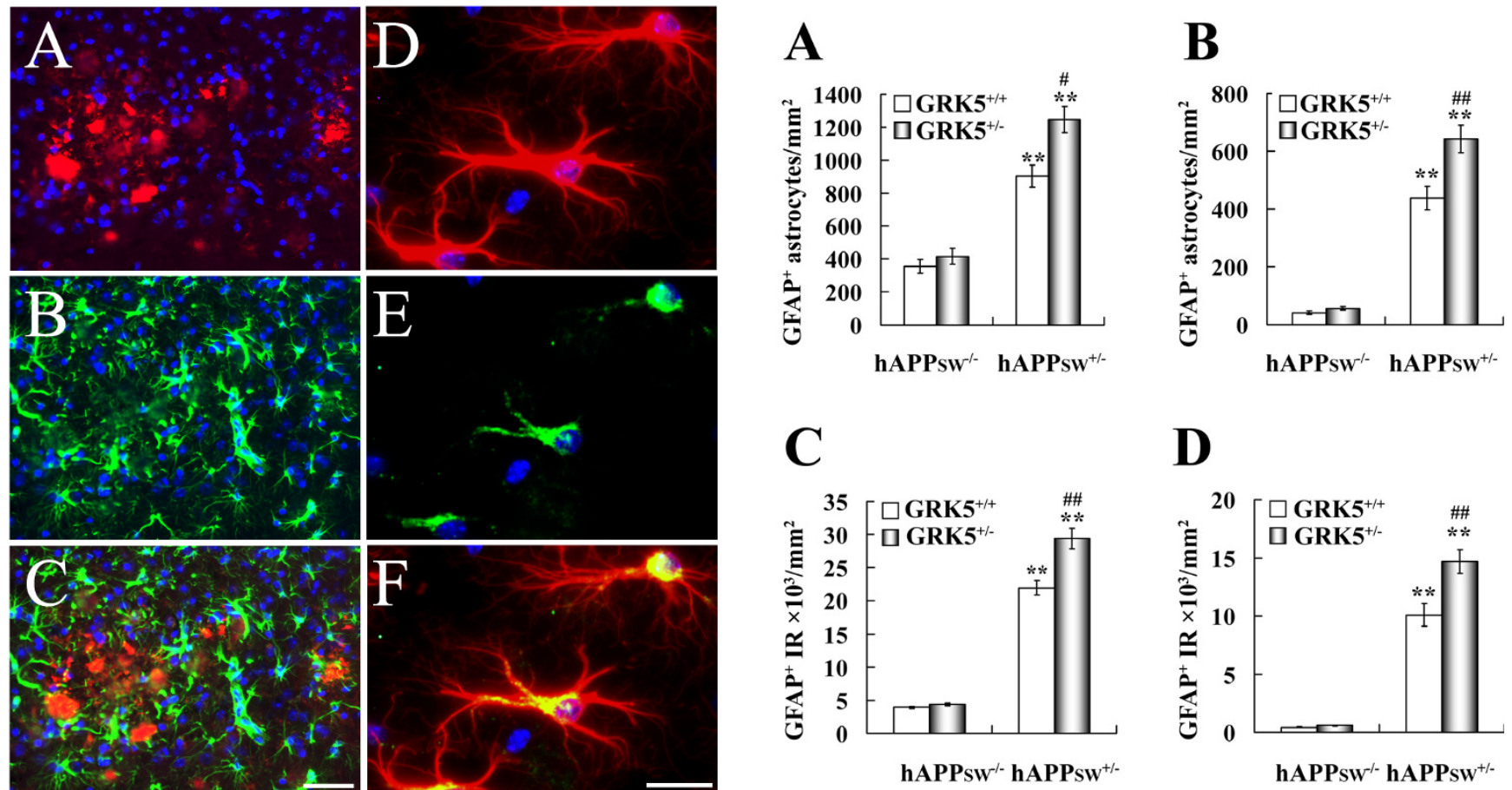

\section{D}

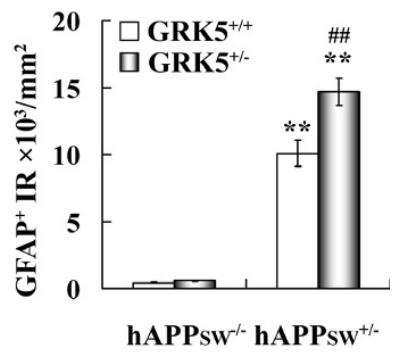

Figure 7

Quantification of GFAP+ astrocytes in hippocampus and cortex of the TgAPPsw mice with reduced GRK5. Both the numbers of GFAP ${ }^{+}$astrocytes (A \&B) and the intensity of GFAP-IR (C \&D) in hippocampus (A \&C) and cortex (B \&D) of WT, heterozygote GRK5KO, TgAPPsw, and the double mice were quantified as described in methods. Two way-ANOVAs revealed significant interactions between APPsw and GRK5 gene modifications with the interaction factors of $p<0.05$ for panel $\mathbf{A}$, and $p<0.01$ for panels B, C, and D. The results for Bonferroni post-hoc comparisons of the means were shown as indicated. **P $<$ 0.001 vs WT or GRK5KO; \#P $<0.01$, \#P $<0.001$ vs TgAPPsw mice.

GRK5 deficiency alone does not lead to any significant inflammatory changes in either the heterozygote or homozygote GRK5KO mice. However, when an inflammation is present, as evoked by overexpression of the Swedish mutated APP gene, the GRK5 deficiency synergistically interacts with the active inflammatory processes and leads to amplified inflammatory responses. Such phenomena are consistent with the manner of GRK action, as GRKs are not thought to induce signals directly, but rather, they primarily modify the activity of activated GPCRs by uncoupling the receptor from G-protein, and initialing receptor internalization $[11,12]$. In the GRK5KO mice, the reason why we did not observe a significant inflammation is likely because there was no an active inflammatory signaling for the GRK5 to act on. In the double mice, however, when Swedish mutated APP was overexpressed, the accumulation of fibrillar $A \beta$ initiated a complex of active inflammatory processes, which provided an opportunity to reveal impact of GRK5 defi- ciency. As demonstrated in this study, inactivation of one copy of the native GRK5 gene led to approximately doubled extent of microgliosis and significantly exaggerated astrogliosis. Therefore, these in vivo results strongly support the model that GRK5 deficiency indeed plays a significant role in amplifying the brain inflammatory responses in $\mathrm{AD}$, as we previously speculated.

Since the primary role of GRKs is to desensitize GPCRs and dampen the activated GPCRs signaling, deficiency of GRK5 may lead to hyperactivity of those GPCRs that are specifically regulated by GRK5. As previously demonstrated, the fibrillar $A \beta$ can initiate a complicated inflammatory process, which may include increased levels of proinflammatory cytokines, chemokines, and activated complement components [1-7]. It is known that receptors for all chemokines and the anaphylatoxin C3a and C5a 
are GPCRs $[6,8,9]$. In fact, even fibrillar $A \beta$ itself has been shown to activate GPCRs, such as macrophage scavenger receptor, or formyl chemotactic receptor 2 (FPR2 or FPRL1) [22-24]. Therefore, theoretically, all these GPCRs could be potential substrates for GRK5. On the other hand, however, the substrate spectrums for different GRK members may overlap, and the lost function due to deficiency of one GRK member may be largely compensated by other GRK members $[11,12]$. Only when the lost function cannot be fully compensated, a phenotype relevant to the specific GPCR signaling can then be revealed. For example, GRK2, GRK5, and GRK6 KO mice have been shown to display selectively impaired desensitization of adrenergic, muscarinic and dopaminergic receptors, respectively $[17,25,26]$. Unfortunately, to the best of our knowledge, information regarding the specific regulation of GRK5 on most, if not all, of the GPCRs involved in the fibrillar $A \beta$ initiated inflammatory processes, particularly the in vivo data, is unavailable. Therefore, further investigations are warranted to understand the detailed molecular mechanisms by which the GRK5 deficiency exaggerates the brain inflammation in the TgAPPsw mice.

The main purpose of this study was to determine whether or not GRK5 deficiency in vivo contributes to exaggerate brain inflammation, and the results confirmed the positive role of GRK5 deficiency in the TgAPPsw mice. In the meantime, the fact that the double mice displayed significantly stronger inflammatory changes than those in the TgAPPsw mice makes the double mice a distinct model from the original TgAPPsw mice. It has been suggested that inflammatory responses in currently available APP transgenic mouse models, including the TgAPPsw mice, are not as strong as those in human $\mathrm{AD}$ patients [16]. Therefore, at least in resembling the strength of the inflammatory responses in $\mathrm{AD}$, the TgAPPsw mice with reduced GRK5 may be a better model than the single TgAPPsw mice, and should serve as an additional AD animal model for relevant studies.

\section{Conclusion}

This study has demonstrated that GRK5 deficiency in vivo significantly exaggerates the microgliosis and astrogliosis in the presence of an inflammatory initiator, the excess fibrillar $\mathrm{A} \beta$ and the subsequent active brain inflammatory reactions in the TgAPPsw mice. In the absence of an active inflammatory response, however, the effects of the GRK5 deficiency on inflammation in vivo remain silent. These findings, along with our previous findings for the role of GRK5 deficiency in promoting axonal defects and synaptic degeneration [13] [Li, 2008, BRB-submitted], strongly support that the hypothesis that GRK5 deficiency plays a significant role in $\mathrm{AD}$ pathogenesis. In addition, the TgAPPsw mouse combined with deficient GRK5 is a better model than the single TgAPPsw mouse, due to a stronger resemblance to the strength of the inflammatory responses in $\mathrm{AD}$.

\section{Abbreviations}

$\mathrm{AD}$ : Alzheimer's disease; $\mathrm{A} \beta$ : $\beta$-amyloid; GPCRs: G-protein coupled receptors; GRK5: GPCR kinase-5; GRK5KO: GRK5 knockout; $\beta$ APP: $\beta$-amyloid precursor protein; PCR: polymerase chain reaction; PBS: phosphate buffered saline; IF: Immunofluorescent; mAb: monoclonal antibody; pAb: polyclonal antibody; GFAP: glial fibrillary acidic protein; IL-1 $\beta$ : interleukin-1beta; IL-6: interleukin6; IR, immunoreactivity.

\section{Competing interests}

The authors declare that they have no competing interests.

\section{Authors' contributions}

LL carried out the pathological studies, performed the statistical analysis, and participated in the maintenance of the transgenic mice and initial drafting of the manuscript, $\mathrm{JL}$ participated in the establishment and maintenance of the transgenic mouse colonies, WZS designed the study, supervised and coordinated the experimental conduction, and helped to draft the manuscript. All authors read and approved the final manuscript.

\section{Acknowledgements}

This study was supported by grants to WZS from the Medical Research and Development Service, Department of Veterans Affairs, the American Federation for Aging Research, and resources from the Midwest Biomedical Research Foundation. In addition, we sincerely thank Drs. Robert J. Lefkowitz and Richard T. Premont at Duke University for providing the original GRK5KO mouse breeding pairs, consultations and review of this manuscript.

\section{References}

I. Wyss-Coray T: Inflammation in Alzheimer disease: driving force, bystander or beneficial response? Nat Med 2006, I2:1005-1015.

2. Griffin WS: Inflammation and neurodegenerative diseases. Am J Clin Nutr 2006, 83:470S-474S.

3. McGeer PL, McGeer EG: Inflammation, autotoxicity and Alzheimer disease. Neurobiol Aging 2001, 22:799-809.

4. van Beek J, Elward K, Gasque P: Activation of complement in the central nervous system: roles in neurodegeneration and neuroprotection. Ann N Y Acad Sci 2003, 992:56-7I.

5. Fiala M, Zhang L, Gan X, Sherry B, Taub D, Graves MC, Hama S, Way D, Weinand M, Witte M, Lorton D, Kuo YM, Roher AE: Amyloidbeta induces chemokine secretion and monocyte migration across a human blood--brain barrier model. Mol Med 1998, 4:480-489.

6. Streit WJ, Conde JR, Harrison JK: Chemokines and Alzheimer's disease. Neurobiol Aging 2001, 22:909-913.

7. Halks-Miller M, Schroeder ML, Haroutunian V, Moenning U, Rossi M, Achim C, Purohit D, Mahmoudi M, Horuk R: CCRI is an early and specific marker of Alzheimer's disease. Ann Neurol 2003 , 54:638-646.

8. Aragay AM, Mellado M, Frade JM, Martin AM, Jimenez-Sainz MC, Martinez AC, Mayor F Jr.: Monocyte chemoattractant protein- Iinduced CCR2B receptor desensitization mediated by the $G$ protein-coupled receptor kinase 2. Proc Natl Acad Sci U S A 1998, 95:2985-2990.

9. Langkabel P, Zwirner J, Oppermann M: Ligand-induced phosphorylation of anaphylatoxin receptors $C 3 \mathrm{aR}$ and $\mathrm{C5} \mathrm{aR}$ is medi- 
ated by "G protein-coupled receptor kinases. Eur J Immunol 1999, 29:3035-3046.

10. Suo Z, Wu M, Citron BA, Wong GT, Festoff BW: Abnormality of G-protein-coupled receptor kinases at prodromal and early stages of Alzheimer's disease: an association with early betaamyloid accumulation. J Neurosci 2004, 24:3444-3452.

II. Kohout TA, Lefkowitz RJ: Regulation of $\mathbf{G}$ protein-coupled receptor kinases and arrestins during receptor desensitization. Mol Pharmacol 2003, 63:9-18.

12. Pitcher JA, Freedman NJ, Lefkowitz RJ: G protein-coupled receptor kinases. Annu Rev Biochem 1998, 67:653-692.

13. Suo Z, Cox AA, Bartelli N, Rasul I, Festoff BW, Premont RT, Arendash GW: GRK5 deficiency leads to early Alzheimer-like pathology and working memory impairment. Neurobiol Aging 2007, 28: 1873-I888

14. Frautschy SA, Yang F, Irrizarry M, Hyman B, Saido TC, Hsiao K, Cole GM: Microglial response to amyloid plaques in APPsw transgenic mice [see comments]. Am J Pathol 1998, I 52:307-3I7.

15. Benzing WC, Wujek JR, Ward EK, Shaffer D, Ashe KH, Younkin SG, Brunden KR: Evidence for glial-mediated inflammation in aged APP(SW) transgenic mice. Neurobiol Aging 1999, 20:58I-589.

16. Schwab C, Hosokawa M, McGeer PL: Transgenic mice overexpressing amyloid beta protein are an incomplete model of Alzheimer disease. Exp Neurol 2004, 188:52-64.

17. Gainetdinov RR, Bohn LM, Walker JK, Laporte SA, Macrae AD, Caron MG, Lefkowitz RJ, Premont RT: Muscarinic supersensitivity and impaired receptor desensitization in $G$ protein-coupled receptor kinase 5-deficient mice. Neuron 1999 24:1029-1036.

18. King DL, Arendash GW, Crawford F, Sterk T, Menendez J, Mullan MJ: Progressive and gender-dependent cognitive impairment in the APP(SW) transgenic mouse model for Alzheimer's disease. Behav Brain Res 1999, 103:145-162.

19. Lee JY, Cole TB, Palmiter RD, Suh SW, Koh JY: Contribution by synaptic zinc to the gender-disparate plaque formation in human Swedish mutant APP transgenic mice. Proc Natl Acad Sci U S A 2002, 99:7705-77।0.

20. Callahan MJ, Lipinski WJ, Bian F, Durham RA, Pack A, Walker LC: Augmented senile plaque load in aged female beta-amyloid precursor protein-transgenic mice. Am J Pathol 200I, 158: II73-1177.

21. Sedgwick JD, Schwender S, Imrich H, Dorries R, Butcher GW, ter Meulen V: Isolation and direct characterization of resident microglial cells from the normal and inflamed central nervous system. Proc Natl Acad Sci U S A 1991, 88:7438-7442.

22. El Khoury J, Hickman SE, Thomas CA, Cao L, Silverstein SC, Loike JD: Scavenger receptor-mediated adhesion of microglia to betaamyloid fibrils. Nature 1996, 382:716-719.

23. Le Y, Gong W, Tiffany HL, Tumanov A, Nedospasov S, Shen W, Dunlop NM, Gao JL, Murphy PM, Oppenheim JJ, Wang JM: Amyloid (beta)42 activates a G-protein-coupled chemoattractant receptor, FPR-like-I. J Neurosci 200I, 2 I:RCI23.

24. Yazawa H, Yu ZX, Takeda, Le Y, Gong W, Ferrans VJ, Oppenheim JJ, Li CC, Wang JM: Beta amyloid peptide (Abeta42) is internalized via the G-protein-coupled receptor FPRLI and forms fibrillar aggregates in macrophages. Faseb J 200I, 15:2454-2462.

25. Jaber M, Koch WJ, Rockman H, Smith B, Bond RA, Sulik KK, Ross J Jr., Lefkowitz RJ, Caron MG, Giros B: Essential role of beta-adrenergic receptor kinase $I$ in cardiac development and function. Proc Natl Acad Sci U S A 1996, 93: I 2974-I 2979.

26. Gainetdinov RR, Bohn LM, Sotnikova TD, Cyr M, Laakso A, Macrae AD, Torres GE, Kim KM, Lefkowitz RJ, Caron MG, Premont RT: Dopaminergic supersensitivity in $\mathrm{g}$ protein-coupled receptor kinase 6-deficient mice. Neuron 2003, 38:291-303.

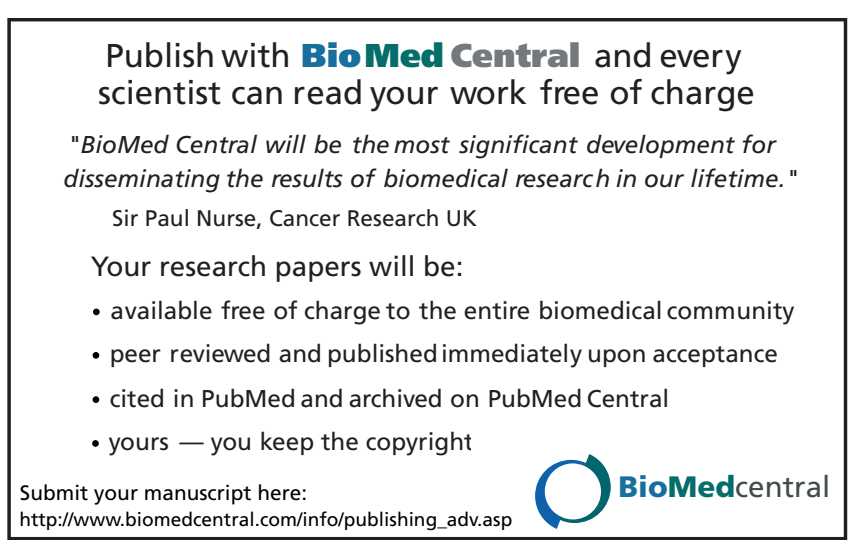

